\title{
DIABETES
}

\section{A role for CFTR in $\beta$-cell function}

CFTR, the cystic fibrosis transmembrane conductance regulator, has a role in insulin secretion by pancreatic $\beta$ cells, indicates recent research.

A substantial proportion of patients with cystic fibrosis have impaired glucose metabolism and many develop diabetes mellitus. Two new studies show that mutations in the gene encoding CFTR, which lead to the development of cystic fibrosis, are also associated with impaired insulin secretion in $\beta$ cells.

The investigators of the latest of these studies observed that CFTR knockout mice have lower insulin levels than wildtype mice, but no obvious destruction of pancreatic tissue. "The reduced levels of insulin in the mice with cystic fibrosis do not result from destruction of $\beta$ cells," says senior author Hsiao Chang Chan.

Using electrophysiology and cell culture techniques as well as experiments with mice carrying the most common CFTR mutation, Chan and co-workers showed that CFTR in $\beta$ cells is important for glucose sensitivity, contributes to the resting membrane potential of $\beta$ cells and mediates action potentials that are coupled to insulin secretion. "These results suggest that CFTR is critical to the normal function of $\beta$ cells, particularly in the first phase of glucose-induced insulin secretion," says Chan.

The researchers also tested the effects of a drug that corrects misfolding of the mutated CFTR protein and prevents its rapid degradation. Application of the drug to cells in culture rescued insulin secretion. Chan hopes that these results will be useful for developing new therapies to treat patients with cystic fibrosis and insulin deficiency.

Joana Osório

Original article Guo, J. H. et al. Glucose-induced electrical activities and insulin secretion in pancreatic islet $\beta$-cells are modulated by CFTR. Nat. Commun. 5 , 4420 (2014)

Further reading Edlund, A. et al. CFTR and Anoctamin 1 (AN01) contribute to cAMP amplified exocytosis and insulin secretion in human and murine pancreatic $\beta$-cells. BMC Med. 12, 87 (2014) 